Revue d'histoire de l'Amérique française

ZRS REVUE D.HISTOIRE DE L'AMÉRIQUE FRANÇAISE

\title{
Le long règne de Duplessis : un essai d'explication
}

\section{René Durocher}

Volume 25, numéro 3, décembre 1971

URI : https://id.erudit.org/iderudit/303095ar

DOI : https://doi.org/10.7202/303095ar

Aller au sommaire du numéro

Éditeur(s)

Institut d'histoire de l'Amérique française

ISSN

0035-2357 (imprimé)

1492-1383 (numérique)

Découvrir la revue

Citer cette note

Durocher, R. (1971). Le long règne de Duplessis : un essai d'explication. Revue d'histoire de l'Amérique française, 25(3), 392-396.

https://doi.org/10.7202/303095ar d'utilisation que vous pouvez consulter en ligne.

https://apropos.erudit.org/fr/usagers/politique-dutilisation/ 


\section{LE LONG REGNE DE DUPLESSIS:} UN ESSAI D'EXPLICATION

\section{ReNÉ DUROcher \\ Département d'histoire York University}

Des vingt-deux premiers ministres que s'est donné le Québec depuis 1867, Maurice Duplessis a été celui qui a détenu ce poste stratégique le plus longtemps, soit dix-huit ans (1936 à 1939 et 1944 à 1959). Comment expliquer ce succès et comment apprécier l'œuvre de ce personnage controversé de notre histoire ?

Personne ne contestera que Duplessis avait une forte personnalité, un réel talent de polémiste et une grande capacité de travail. On peut même lui reconnaître un certain charisme 
auprès des foules. Pourtant, malgré tout, l'homme n'est pas très impressionnant. A moins de confondre la mémoire, l'art du calembour, la ruse et l'opportunisme avec l'intelligence, on ne saurait considérer Duplessis comme un homme exceptionnellement doué sur le plan intellectuel. De plus, sa culture et son imagination m'apparaissent assez étroites.

A travers ses écrits, ses déclarations, ses discours et les mémoires d'hommes politiques qui l'ont bien connu - comme René Chaloult, Georges-Emile Lapalme, Antonio Barrette, Pierre Laporte - on peut ébaucher un premier portrait de l'homme. Cependant, pour que l'esquisse soit un peu moins grossière, il nous faudrait interroger des gens de son entourage. Il nous faudrait aussi ses papiers personnels qui nous éclaireraient grandement comme j'ai eu l'occasion de le constater en dépouillant sa correspondance avec Mitchell Hepburn et Mackenzie King pour les années 1936-1939. Espérons que la famille Duplessis conserve précieusement ce fonds documentaire et que dans les meilleurs délais elle saura le mettre à la disposition des chercheurs en le confiant aux Archives du Québec.

Même si on pouvait faire la biographie de Duplessis et même si on démontrait qu'il avait une personnalité et une intelligence absolument exceptionnelles, il est fort raisonnable de croire que si Duplessis était resté chef du parti conservateur provincial, il n'aurait guère plus d'importance dans notre histoire que des hommes comme Mathias Tellier, Evariste Leblanc, Philémon Cousineau ou Arthur Sauvé. Dans notre système politique, on n'accède au pouvoir qu'au moyen d'un parti politique et on n'y reste qu'à condition de bien le dominer.

N'importe quel parti ne peut convenir. Si, par exemple, Pierre-Elliott Trudeau était resté fidèle au NPD, non seulement il ne serait pas premier ministre du Canada mais il ne serait même pas député. De même, Duplessis a su choisir le "bon" parti au moment propice. Il a été un remarquable chef de parti et l'Union Nationale a été, pour lui, un instrument extraordinairement efficace pour prendre puis conserver le pouvoir. L'étude de ce parti en tant que parti reste à faire malgré l'honnête tentative de Quinn.

Il convient de souligner dans une telle rechercre la remarquable stabilité politique du Québec de 1897 à 1959, qui contraste avec la période de 1867-1896 et avec celle de 1960-1971. Il ne fait pas de doute que le système politique que nous avons a contribué à cette stabilité. Il est probable aussi que la nouvelle 
industrialisation du Québec, qui s'amorce au début du XX $\mathrm{XX}^{\mathrm{e}}$ siècle, soit l'une des causes de ces longs règnes de quinze ans et plus: Gouin, Taschereau et Duplessis.

L'étude comparée de ces trois régimes que je viens de mentionner est d'ailleurs fort intéressante. L'on ne saurait nier que dans les trois cas le système électoral, la carte électorale fortement biaisée en faveur des comtés ruraux et la législation électorale favorisaient nettement le parti au pouvoir sauf dans des situations exceptionnelles comme la crise économique ou la guerre de '39. De même le patronage, c'est-à-dire l'appropriation par le parti et pour ses fins des pouvoirs et des fonds de l'Etat, a permis à ces trois régimes de se maintenir longtemps au pouvoir. Soulignons que le patronage, c'est infiniment plus que la caisse électorale qui n'en est qu'un aspect limité et peut-être pas le plus important.

Dans plusieurs comtés où la lutte était très serrée entre les adversaires, ce facteur patronage, pris dans son sens large, pouvait être déterminant. Plusieurs ont tendance à minimiser ce facteur politique au profit d'une explication idéologique: c'est-àdire le nationalisme de Duplessis qui s'exprime dans sa conception de l'autonomie provinciale. Certains ne sont pas loin de penser que le succès de Duplessis c'est, d'abord et avant tout, l'accord profond entre le nationalisme autonomiste de Duplessis et la population québécoise.

Les nombreuses recherches sur les idéologies qui ont été faites par des sociologues, des politicologues et des historiens nous permettent de mieux voir, peu à peu, que l'idéologie de la petite bourgeoisie canadienne-française a été l'idéologie dominante de 1840 à 1960 . Il ne me paraît guère douteux que l'idéologie de Duplessis correspondait assez fidèlement à cette idéologie dominante essentiellement conservatrice et cléricale et que c'est une des causes de son succès. Cette idéologie prend tout son sens comme explication si on ne la réduit pas à l'autonomie provinciale et si l'on évite de considérer le peuple canadien-français comme une espèce de classe ethnique. Il faut relier l'idéologie duplessiste aux classes sociales et aux structures économiques du Québec de cette époque. C'est ainsi qu'on pourrait donner tout son sens à la théorie du roi-nègre de André Laurendeau. C'est ainsi qu'on comprendrait comment Duplessis, en même temps qu'il chante les vertus de l'agriculture, préside à un certain développement industriel axé sur les ressources naturelles. On comprendrait mieux aussi comment ce défenseur de notre "butin", 
c'est-à-dire de notre autonomie provinciale, cède des parties du territoire québécois à l'étranger... .

A toutes ces causes que j'ai mentionnées pour expliquer le succès de Duplessis, il faut ajouter la conjoncture politique et économique spécifique de cette époque, qui le favorisait singulièrement. En 1936, par exemple, c'est grâce pour une bonne part, au travail de l'Action libérale nationale et à l'impact de la crise économique qu'il a réussi à accéder au pouvoir. En 1939, comme en 1944, la conscription joue un rôle majeur dans sa défaite comme dans sa victoire. En 1948, 1952 et 1956 le Québec connaît une extraordinaire période de croissance économique dont Duplessis profite largement. A propos des années 19471956, l'économiste Gilles Lebel écrit: "Durant toute cette période, le Québec a continué de tirer parti des principaux facteurs de sa croissance économique d'après-guerre: la croissance rapide de sa population de $2.5 \%$ par an, l'exploitation de ses ressources, $13 \%$ d'augmentation annuelle dans les investissements des secteurs primaires et de la construction, une main-d'œuvre employée à environ $97 \%$, et la demande mondiale continue qui avantageait l'exploitation d'un grand nombre de nos denrées de base." 1 Notons aussi que si l'on veut apprécier l'action du régime duplessiste, il faut tenir compte de cette conjoncture économique hautement favorable et non pas se contenter d'additionner, comme lui-même le faisait, les ponts, les hôpitaux et les écoles qu'il a fait construire de 1944 à 1959.

Pendant que Duplessis se préoccupait de l'agriculture ou pourfendait les étrangers, l'entreprise privée assurait l'expansion économique. Pendant qu'il dénonçait le gouvernement fédéral, celui-ci développait une modeste politique sociale qui correspondait aux exigences d'une société industrielle et urbanisée. Dans un cas comme dans l'autre, Duplessis en bénéficiait car la population se montrait moins exigeante pour son gouvernement provincial. Il a eu la chance de diriger une population qui avait été traumatisée par la crise et par la guerre. Les Québécois avaient des aspirations modestes que l'expansion économique et la politique fédérale arrivaient à combler. De plus, la guerre avait affaibli, et pour longtemps, le parti libéral provincial qui, en plus d'être écrasé par son homonyme fédéral, a manqué de leadership et de clairvoyance, en particulier sur la question de l'autonomie provinciale.

${ }^{1}$ Gilles Lebel, Horizon 1980: une étude sur l'évolution de l'économie du Québec de 1946 à 1968 et sur ses perspectives d'avenir (Québec, ministère de l'Industrie et du Commerce, 1970), 91. 
Malgré toutes ces conditions extrêmement favorables, c'est de justesse qu'il prend et conserve le pouvoir. En effet, en 1944, il n'a que $35.8 \%$ des suffrages populaires pour s'emparer du pouvoir; en 1948 - qui a été l'année de sa grande victoire, en nombre de députés - il n'a que $51 \%$ des votes; en 1952 et en 1956, il n'obtient que $51.5 \%$ et $52 \%$ des votes. Devant ces chiffres, on peut se demander si on n'a pas exagéré la force et la représentativité de Duplessis.

Même s'il a pu freiner le développement du Québec, il n'a pu empêcher tout changement. Pendant qu'il glorifiait le passé, les traditions et l'agriculture, des réalités nouvelles investissaient le Québec. Après sa mort, un Québec en gestation depuis quinze ans a fait la révolution tranquille. Au nationalisme conservateur et réactionnaire de Duplessis a succédé un nouveau nationalisme tourné vers l'avenir et le progrès social. 